\title{
Design and realization of future compact external-cavity diode laser systems
}

\author{
S. Baumgärtner*, A. Matteo, N. Kolodzie, C. Nölleke, C. Raab and P. Leisching, \\ TOPTICA Photonics AG, Lochhamer Schlag 19, 82166 Gräfelfing, Germany
}

\begin{abstract}
Copyright 2019 Society of Photo-Optical Instrumentation Engineers (SPIE). One print or electronic copy may be made for personal use only. Systematic reproduction and distribution, duplication of any material in this paper for a fee or for commercial purposes, or modification of the content of the paper are prohibited.
\end{abstract}

DOI: https://doi.org/10.1117/12.2509620

Cite as: S. Baumgärtner, A. Matteo, N. Kolodzie, C. Nölleke, C. Raab, and P. Leisching "Design and realization of future compact external-cavity diode laser systems", Proc. SPIE 10899, Components and Packaging for Laser Systems V, 108990D (4 March 2019); https://doi.org/10.1117/12.2509620

\begin{abstract}
Compact and robust external-cavity diode laser (ECDL) systems are a mandatory requirement for many next-generation quantum technology applications, e.g. quantum communication and quantum sensors. Today's commercially available ECDLs are used for proof-of-principle demonstrations of such applications, however do not meet the requirements for the use in real-world environments.

We investigate a novel design for a compact and robust ECDL suitable for the integration into first quantum technology applications. Experimental results of first prototypes are presented and compared to a commercially available ECDL and numerical simulations.
\end{abstract}

Keywords: diode laser, ECDL, miniaturization

\section{INTRODUCTION}

Quantum technology (QT) will play a decisive role for future advances in our everyday life. Worldwide, several large and ambitious research initiatives have recently been started or are currently being planned in order to transition these new technologies from research laboratories into real-world applications. The European Union is actively supporting quantum technologies within the Horizon 2020 program [1] and the quantum technologies flagship [2]. They focus on four application areas: quantum communication, quantum computing, quantum simulation and quantum metrology and sensing. Examples for funded projects are QSource [3] - a project to develop scalable single-photon sources for quantum key distribution, AQTION [4] - which aims to realize a scalable quantum computer, ColOpt [5] - where collective effects in cold atoms are studied and iqClock [6] - a project devoted towards a transportable, simple-to-use and affordable optical clock. Most of these applications require specialized laser systems with high demands on wavelength coverage, output power, spectral purity and stability. For example, the AQTION project requires a laser which linewidth's can be brought down to $1 \mathrm{~Hz}$, while the ColOpt project requires a single-frequency laser with a high output power of several Watts. For the use in research laboratories such systems based on diode- and solid-state lasers are readily available. However, the aforementioned projects require the lasers to be compact turnkey systems that can be easily integrated into the QT system, while at the same time being mechanically robust. Single-frequency diode lasers are the most promising option to satisfy the requirements of the QT initiatives. In general, diode lasers can be operated without complex pump or cooling setups and have a high wall-plug efficiency. This allows diode-laser systems to be miniaturized and hence be integrated into portable quantum sensors such as gravimeters and magnetometers. 
To achieve single-mode operation in a diode laser, controlled optical feedback into the diode is required. Distributed feedback (DFB) and distributed Bragg-reflector (DBR) diodes use grating structures inside the semiconductor chip to generate optical feedback, making these devices insensitive to acoustic noise. However the production of DFB- and DBR-diodes is a sophisticated and expensive process and the commercially available wavelength range is currently limited down to $626 \mathrm{~nm}[7,8]$. Furthermore the typical linewidth of DFB- and DBR-diodes is around $1 \mathrm{MHz}$ and therefore not small enough for many QT applications. External-cavity diode lasers (ECDL) use optical feedback from an external element. Using ECDLs, a laser linewidth in the range of $1 \mathrm{kHz}$ can be achieved. By means of an active stabilization to a high-finesse cavity, linewidths down to the range of $1 \mathrm{~Hz}$ have been realized [9]. Probably the best known realizations of an ECDL are the designs proposed by Littrow and Littman [10, 11], which are based on feedback from a reflection grating. The grating serves as a frequency-selective element and controls the emission frequency. Other components can also be used as frequency-selective elements, e.g. transmission gratings [12], fiber Bragg-gratings [13], volume Bragg-gratings (VBG) [14], bandpass filters [15], on-chip waveguides with integrated gratings [16] and micro resonators [17] have already been used to realize an ECDL. All these implementations of ECDLs have different advantages. In general, trade-offs exist between different system properties such as output power, linewidth, tunability, size, stability and the achievable wavelength region The designs using only components integrated on the same submount like on-chip waveguide and micro-resonators [16, 17] are robust and insensitive to acoustic noise. However, producing these devices require sophisticated and expensive manufacturing steps which are limited to certain wavelength regions. Designs using macroscopic optical components such as VBGs or filters result in a larger resonator setup which are more susceptible to acoustic noise, however are more flexible and typically result in higher output powers and smaller linewidths.

This work reports on an ECDL which is based on optical feedback from a partial-reflective mirror and a bandpass filter as the frequency-selective element. The resonator design is simple, while at the same time all components are commercially available. Hence this design enables the realization of a compact laser system which at the same time is flexible in terms of output power and available wavelengths.

\section{ECDL DESIGN \& OPTICAL PERFORMANCE}

A schematic of the laser setup is shown in Fig. 1. The ECDL consists of a laser diode emitting around $789 \mathrm{~nm}$, a collimation lens, the bandpass filter and a partially reflective mirror to form the external resonator.

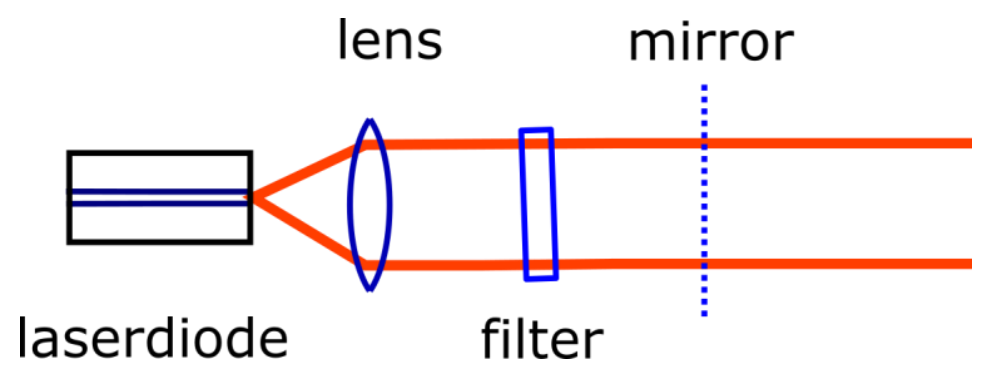

Figure 1 Schematic of the ECDL design. The ECDL consists of a laser diode, a collimation lens, the bandpass filter as frequencyselective element and a partially reflective mirror to form the external resonator. The emission frequency can be tuned by rotating the filter and by changing the internal and external modes.

Compared to a Littrow ECDL the losses are increased due to the filter inside the cavity. Also the bandwidth of the filter for the frequency selection is typically larger than that of a grating. In contrast, the filter design is more flexible because the frequency selection and the optical feedback are separated and can be controlled independently. Furthermore there is no beam displacement during wavelength tuning. The emission frequency of the laser can be coarsely controlled by rotating the filter. Additionally the internal and external modes can be shifted to fine tune the emission frequency. All 
optical components were actively aligned and mounted to the optical bench. During the initial alignment we could set and maintain the emission frequency within an uncertainty of $50 \mathrm{GHz}$.

In the following, we characterize the optical performance of the prototype. In Fig. 2, the current-power characteristics is shown with a maximum power of over $140 \mathrm{~mW}$ at a wavelength of $789 \mathrm{~nm}$. The power jumps are attributed to mode hops. The current-power characteristic and the output power are comparable to commercially available Littrow-based ECDLs [18] with the same laser diode from eagleyard Photonics [8].

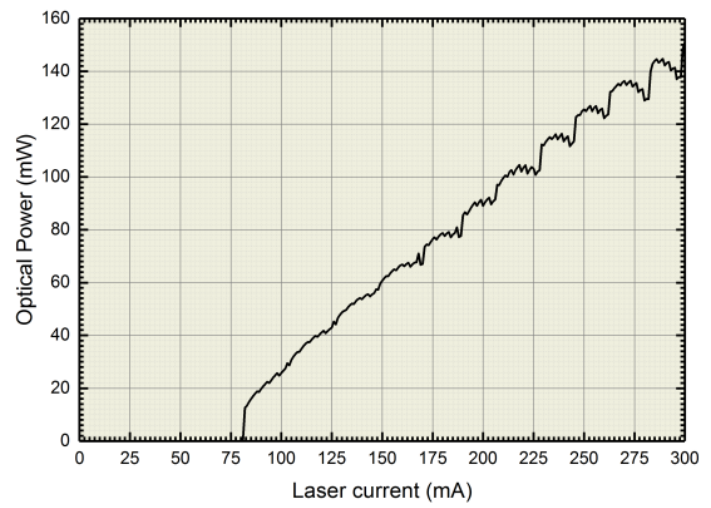

Figure 2: Current-power characteristic of the laser module. The laser threshold is at $81 \mathrm{~mA}$ for an operating temperature of $23{ }^{\circ} \mathrm{C}$.

The corresponding optical spectrum is shown in Fig. 3. It exhibits a single peak which width is limited by the resolution of the optical spectral analyzer (OSA). The side-mode suppression is $>70 \mathrm{~dB}$ and is limited by the dynamic range of the OSA.

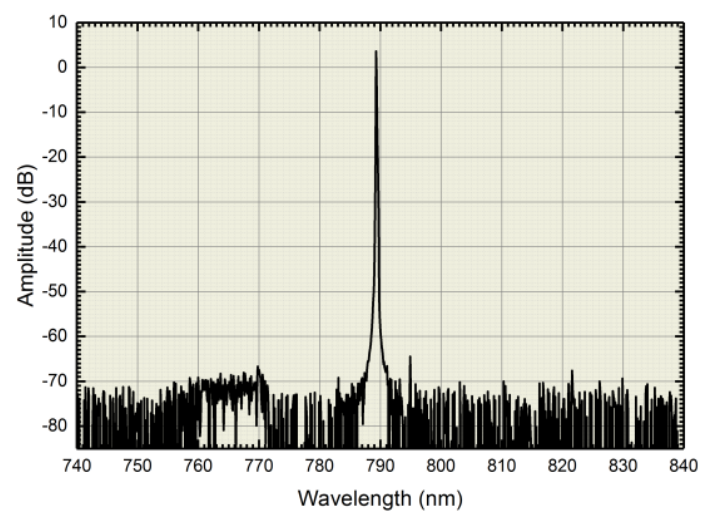

Figure 3: Spectrum of the laser module measured at $23^{\circ} \mathrm{C}$ and maximum current of $300 \mathrm{~mA}$.

The beam profile was also recorded for the same laser conditions and is shown in Fig. 4. Measuring the $\mathrm{M}^{2}$ characteristic the laser reveals values of $\mathrm{M}_{\mathrm{x}}^{2}=1.3$ and $\mathrm{M}_{\mathrm{y}}^{2}=1.1$. The difference in both axes is caused by different divergences of the fast and slow axis of the laser diode. The collimation with a single lens results in an elliptical beam profile. Using an additional cylindrical lens or an anamorphic prism pair a round beam profile can be achieved. The coupling efficiency into a single-mode fiber is typically $>70 \%$. 


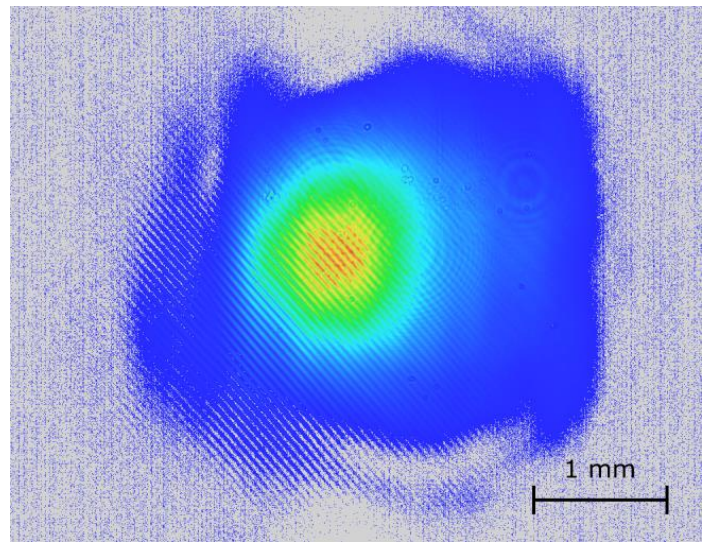

Figure 4: Beam profile of the laser module measured with a camera for a current of $300 \mathrm{~mA}$ and a temperature of $23{ }^{\circ} \mathrm{C}$.

Furthermore the power spectral density of the frequency noise of the laser was measured using a self-heterodyne technique [19] (Fig. 5).

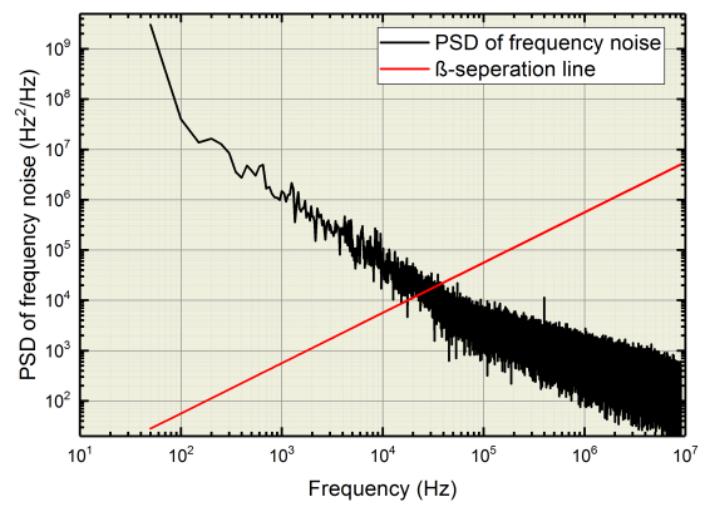

Figure 5: Power spectral density of the frequency noise of the laser module.

With the information from the power spectral density of the frequency-noise the linewidth can be calculated. Using the approach from [20], the estimated linewidth is $200 \mathrm{kHz}$ for an observation time of $5 \mathrm{~ms}$. The Lorentzian linewidth is estimated to be less than $5 \mathrm{kHz}$. The noise characteristic is comparable to commercially available ECDLs based on reflection gratings. Hence the laser will allow state-of the art frequency-locking schemes to be implemented as required for quantum technology applications.

The emission frequency of the laser can be tuned by varying the filter angle and the internal and external modes. For frequency stabilization, e.g. to a reference cavity or to a frequency comb single-frequency operation and a large modehop free tuning range is mandatory. In Fig. 6, a so called modemap measurement is shown [21]. For this measurement the length of the internal and the external resonator is varied, while recording the emission wavelength of the laser using a wavelength meter from High Finesse with an accuracy of $1 \mathrm{pm}$. At the same time, it is measured if the laser is operating on a single-longitudinal-mode. The emission wavelength is depicted in false colors. 

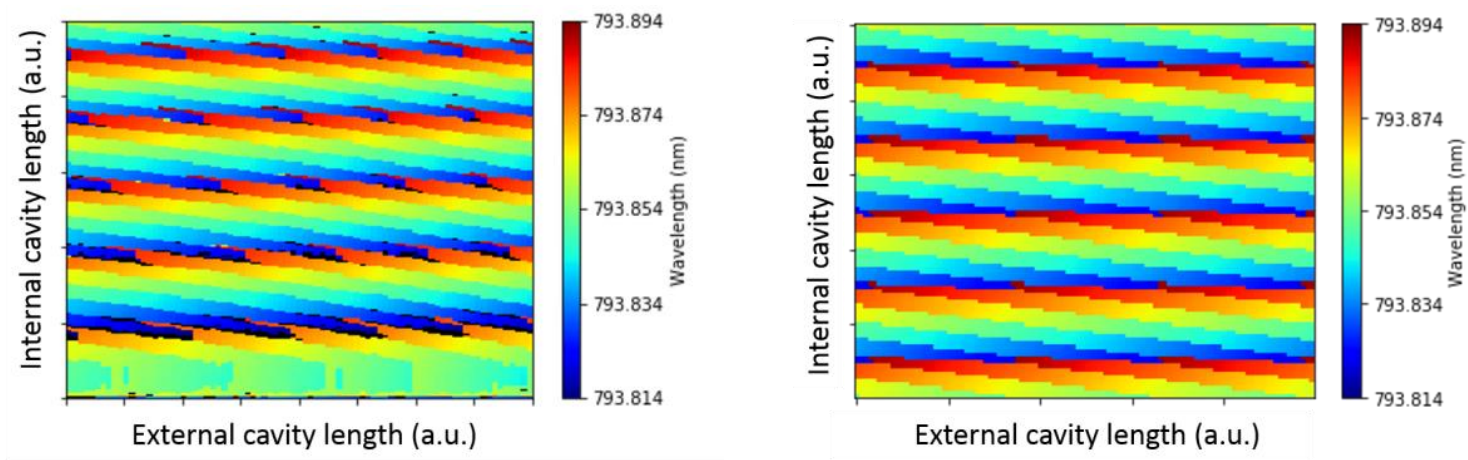

Figure 6: Modemap of the laser as a function of the internal and external resonator length. The left picture shows the measurement, the right picture the simulation result.

The laser is emitting a single longitudinal-mode over the whole operating range of the laser parameters. Changing only the internal or the external resonator results in a mode-hop-free tuning range (MHFTR) of a few GHz. The measured mode hops are periodically with respect to the internal and external cavity length, respectively. By appropriately tuning the internal and external resonator simultaneously the MHFTR can be maximized. By this means a MHFTR of $25 \mathrm{GHz}$ can be achieved.

A simulation of the modemap is shown in Fig. 6. The simulation is based on calculating the theoretically expected emission wavelength for each pair of values for the internal and external cavity length, while taking into account the parameters of the filter and mirror. The simulation is in very good agreement with the measurement results. The same periodicity as in the measurement can be observed. We attribute the minor differences of the simulation to the measurement to hysteresis and other nonlinear effects which are not taken into account in the simulation. These kind of simulations will enable us to optimize the design of the optical resonator with respect to specific quantum technology applications.

\section{ENVIROMENTAL TEST}

Some QT applications require the laser system to be operated in harsh environments and therefore have a particular demand on reliability and lifetime. For example, quantum key distribution over long-distances requires the single-photon source to be installed on a satellite in orbit $[3,22]$. For such applications where robustness and stability are of paramount importance, the HALT and HASS methodology is widely used in industry [23].

As a first step towards complete HASS and HALT measurements and to test the stability, we exposed an ECDL to changing environmental conditions and then measured after each test. The threshold is sensitive to changes in the alignment of the laser and it therefore serves as a simple measure, of the system's overall stability. As an example several measurements of the threshold and maximum power are shown in Fig. 8 for different environmental conditions. The first measurement is conducted with the laser directly after initial alignment. The following measurements are normalized to this value. When the laser is exposed to temperature changes between $-5^{\circ} \mathrm{C}$ and $+45^{\circ} \mathrm{C}$, only small effects of less than $\pm 5 \%$ on threshold and output power can be observed. Most importantly, the laser is still emitting a single longitudinal-mode. 


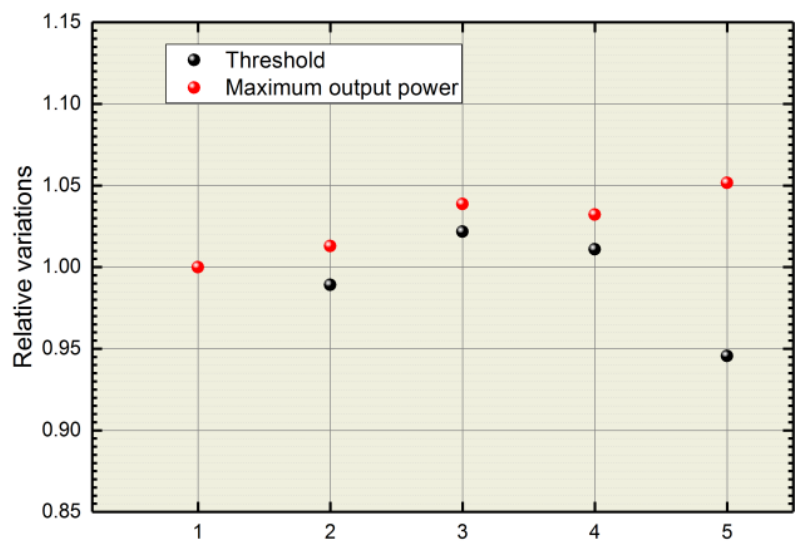

\begin{tabular}{|c|c|}
\hline $\begin{array}{c}\text { Measurement } \\
\text { ID }\end{array}$ & Description \\
\hline 1 & Initial alignment \\
\hline 2 & Shelf storage test \\
\hline 3 & $45^{\circ} \mathrm{C}$ stress test \\
\hline 4 & Shelf storage test \\
\hline 5 & $-5^{\circ} \mathrm{C}$ stress test \\
\hline
\end{tabular}

Figure 8: Relative changes of the threshold and the output power of the laser for different environmental tests. The results of the individual measurements are normalized to the threshold and power directly after initial alignment.

A second laser module with the same design shows a similar behavior. Future investigations will be done to extend the maximum temperature range and investigate the impact of vibrations during transport, storage and transportation.

\section{CONCLUSION}

We have presented a novel design and analysis for a compact and robust ECDL laser system for future quantum technology applications. The maximum output power is $140 \mathrm{~mW}$ while the side-mode suppression is $>70 \mathrm{~dB}$. Furthermore the beam profile was measured with $\mathrm{M}^{2}$ values of $\mathrm{M}^{2}=1.3$ and $\mathrm{M}_{\mathrm{y}}^{2}=1.1$ and fiber coupling efficiency $>70 \%$. The linewidth was calculated from the measured frequency-noise density to be $200 \mathrm{kHz}$. The laser is tunable with a maximum MHFTR of $25 \mathrm{GHz}$ while it is emitting a single longitudinal-mode over the whole operating range. Environmental tests between $-5{ }^{\circ} \mathrm{C}$ and $45{ }^{\circ} \mathrm{C}$ show the basic suitability of the design for a compact and robust ECDL. These tests are the first step towards a complete HASS and HALT characterization of the laser module which are especially important for industrial applications. Future characterization will also include tests in accordance with standards like MIL-STD-810.

\section{ACKNOWLEDGEMENTS}

TOPTICA's work on this project was supported by the German Federal Ministry of Research (BMBF) by a grant under program QSource (FKZ: 13N14978) and the European Union Horizon 2020 Research and innovation program under the Marie Sklodowska-Curie grant no. 721465 named ColOpt.

\section{REFERENCES}

[1] https://ec.europa.eu/programmes/horizon2020/en

[2] Riedel, M, Binosi, D., Thew, R. and Calarco, T. "The European quantum technologies flagship programme." Quantum Science and Technology, vol. 2, no. 3, 030501 (2017).

[3] https://www.photonikforschung.de/projekte/quantentechnologien/projekt/qsource.html

[4] https://ec.europa.eu/digital-single-market/en/content/aqtion-advanced-quantum-computing-trapped-ions

[5] https://www.colopt.eu/

[6] https://ec.europa.eu/digital-single-market/en/content/iqclock-time-telling-ultra-precision 
[7] Blume, G., Nedow, O., Feise, D., Pohl, J., and Paschke, K. "Monolithic single-mode AlGaInP DBR diode laser”, Opt. Express, Vol. 21, no. 18, 21677-21684 (2013).

[8] https://www.eagleyard.com/products/single-frequency-laser-diodes/

[9] Stoehr, H., Mensing, F., Helmcke, J. and Sterr, U. "Diode laser with 1 Hz linewidth." Optics letters“, vol. 31, no. 6, 736-738 (2006).

[10] Ricci, L., Weidemüller, M., Esslinger, T., Hemmerich, A., Zimmermann, C., Vuletic, V., König, W. and T.W. Hänsch, "A compact grating-stabilized diode laser system for atomic physics", Opt. Comm., Vol. 117, no. 5, 541-549, (1995).

[11]Liu, K. and Littman, M. G., "Novel geometry for single-mode scanning of tunable lasers", Opt. Lett., Vol. 6, no. 3, 117-118, (1981).

[12] Laurila, T., Joutsenoja, T., Hernberg, R. and Kuittinen, M. “Tunable external-cavity diode laser at $650 \mathrm{~nm}$ based on a transmission diffraction grating”, Appl. Opt., vol. 41, no. 27, 5632-5637 (2002).

[13] Monica K. Davis and Alina Echavarria, "Polarization-Induced Feedback Effects in Fiber Bragg GratingStabilized Diode Lasers", J. Lightwave Technol., Vol. 22, no. 12, 2816, (2004).

[14] T. Hieta and M. Vainio and C. Moser and E. Ikonen, "External-cavity lasers based on a volume holographic grating at normal incidence for spectroscopy in the visible range", Opt. Comm., Vol. 282, no. 15, 3119-3123 (2009).

[15] Baillard, X., Gauguet, A., Bize, S., Lemonde, P., Laurent, P., Clairon, A. and Rosenbusch, "Interference-filterstabilized external-cavity diode lasers", Opt. Comm., Vol. 266, no. 2, 609-613, (2006).

[16] http://www.rio-lasers.com/

[17] Kondratiev, N. M., Lobanov, V. E., Cherenkov, A. V., Voloshin, A. A., Pavlov, N. G., Koptyaev, S., Gorodetsky, M. L., "Self injection locking of a laser diode to a high-Q WGM microresonator", Opt. Expr., Vol. 25, no. 23, 28167-28178 (2017).

[18] https://www.toptica.com/products/tunable-diode-lasers/ecdl-dfb-lasers/dl-pro/

[19] Tscuhida, H., "Laser frequency modulation noise measurement by recirculating delayed self-heterodyne method", Opt. Letters, vol. 36, no. 5, 681-683 (2011).

[20] Di Domenico, G., Schilt, S., Thomann, P., "Simple approach to the relation between laser frequency noise and laser line shape", Appl. Optics., vol 49, no. 25, 4801-4807 (2010).

[21] Nölleke, C., Leisching, P., Blume, G., Jedrzejczyk, D., Pohl, J., Feise, D., Sahm, A., Paschke, K., „Frequency locking of compact laserdiode modules at 633 nm", Proc. SPIE 10539, 1053907 (2018).

[22] Yin, J., Cao, Y., Li, Y., Liao, S., Zhang, L., ren, J., Cai, W., Liu, W., Li, B., Dai, H., Li, G., Lu, Q., Gong, Y., Xu, L., Li, S., Li, F., Yin, Y., Jiang, Z., Li, M., Jia, J., ren, G., He, H., Zhou, Y., Zhang, X., Wang, N., Chang, X., Zhu, Z., Liu, N., Chen, Y., Lu, C., Shu, R., Peng, C., Wang, J. and Pan, J., "Satellite-based entanglement distribution over 1200 kilometers", vol. 356, no. 6343, 1140-1144 (2017).

[23] McLean, H. W., HALT, HASS, and HASA explained: accelerated reliability techniques. ASQ Quality press, 2009. 